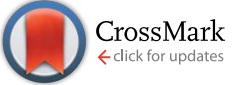

Cite this: RSC Adv., 2016, 6, 21235

Received 22nd December 2015 Accepted 4th February 2016

DOI: $10.1039 / c 5 \mathrm{ra} 27452 \mathrm{~d}$

www.rsc.org/advances

\section{Quality of graphene on sapphire: long-range order from helium diffraction versus lattice defects from Raman spectroscopy $\dagger$}

\author{
Gloria Anemone, ${ }^{a}$ Esteban Climent-Pascual, ${ }^{\text {b }}$ Hak Ki Yu, ${ }^{\text {cd }}$ Amjad Al Taleb, ${ }^{a}$ \\ Felix Jiménez-Villacorta, ${ }^{\mathrm{b}}$ Carlos Prieto, ${ }^{\mathrm{b}}$ Alec M. Wodtke, ${ }^{\text {cd }}$ Alicia De Andrés ${ }^{\mathrm{b}}$ \\ and Daniel Farías*aef
}

\begin{abstract}
We report a new method to produce high-quality, transparent graphene/sapphire samples, using $\mathrm{Cu}$ as a catalyst. The starting point is a high-quality graphene layer prepared by $\mathrm{CVD}$ on $\mathrm{Cu}(111) / \mathrm{Al}_{2} \mathrm{O}_{3}$. Graphene on sapphire is obtained in situ by evaporation of the Cu film in UHV. He-diffraction, atomic force microscopy (AFM), Raman spectroscopy and optical transmission have been used to assess the quality of graphene in a metal free area. We used helium atom scattering as a sensitive probe of the crystallinity of the graphene on sapphire. The observation of high reflectivity and clear diffraction peaks demonstrates the presence of flat and homogeneous graphene domains over lateral scales of microns, consistent with the AFM results. Surprisingly, putting graphene on sapphire improves the quality of the He-diffraction spectra. Graphene forms a moire pattern with a $(11 \times 11)$ periodicity, aligned with the $(1 \times$ 1) sapphire unit cell. The lattice constant of graphene on sapphire is $a=(2.44 \pm 0.02) \AA$. The phonon dispersion of the graphene flexural mode has been measured. This allowed the determination of the bending rigidity $k=0.61 \pm 0.15 \mathrm{eV}$, and the graphene-sapphire coupling strength $g=(5.8 \pm 0.4) \times 10^{19}$ $\mathrm{N} \mathrm{m}^{-3}$. The uniformity of the graphene has also been investigated by Raman mapping. Judging by the ratio of the $2 \mathrm{D}$ to $\mathrm{G}$ peaks, the quality of the graphene is not degraded by $\mathrm{Cu}$ removal. The high transparency $(80 \%)$ measured in the visible range makes this system suitable for many applications that require hybrid properties commonly associated with metals (conductivity) and insulators (transparency). Our study shows that He-diffraction and Raman provide crucial information on quite different, complementary aspects of the same samples.
\end{abstract}

\section{Introduction}

Presently, great effort is being expended to find a suitable replacement for the industry standard transparent conductors in use. This effort is motivated by the fact that these materials require scarce metals (like in indium tin oxide, ITO), perform poorly on flexible substrates and require expensive vacuum deposition techniques. ${ }^{\mathbf{1} 2}$ Reducing the cost of transparent conductors, moving away from elements that are expensive and/ or in short supply, is crucial for the future. ${ }^{3,4}$ Graphene/sapphire

\footnotetext{
a Departamento de Física de la Materia Condensada, Universidad Autónoma de Madrid, 28049 Madrid, Spain.E-mail: daniel.farias@uam.es

${ }^{b}$ Instituto de Ciencia de Materiales de Madrid, Consejo Superior de Investigaciones Cientificas, Spain

'Institute for Physical Chemistry, University of Göttingen, 37077 Göttingen, Germany ${ }^{d}$ Max Planck Institute for Biophysical Chemistry, 37077 Göttingen, Germany

eInstituto "Nicolás Cabrera", Universidad Autónoma de Madrid, 28049 Madrid, Spain ${ }^{f}$ Condensed Matter Physics Center (IFIMAC), Universidad Autónoma de Madrid, 28049 Madrid, Spain
}

$\dagger$ Electronic supplementary information (ESI) available. See DOI: $10.1039 / \mathrm{c} 5 \mathrm{ra} 27452 \mathrm{~d}$ samples may provide a possible alternative to these materials for many applications. However, most of the available methods for controlled and scalable preparation of graphene (Gr) on metallic substrates cannot be easily extended to grow $\mathrm{Gr}$ on sapphire. The standard method to obtain Gr is by chemical vapor deposition (CVD) on metallic foils, mostly copper and nickel, which serve as catalysts for the dissociation of the precursor gas (typically methane or ethylene) to form the $\mathrm{Gr}$ layer. An important drawback is the necessity to eliminate the metallic foil before transferring the Gr layer to substrates adequate for its implementation in devices, in general either transparent substrates or silicon. The metal elimination is performed by using acids, which leads to the appearance of residues in the Gr layer. So far, most of the methods reported have not produced Gr layers of quality comparable to that obtained for instance on $\mathrm{Cu}^{5-9}$ Typically, the Raman D-peak (related to the presence of defects) is larger than the G-peak, indicating small grain size $(\sim 15 \mathrm{~nm})$. For $\mathrm{Gr}$ on sapphire, a few methods have been reported in which the Raman D-peak was significantly reduced; these methods are either based on the high-temperature $\left(1500-1600{ }^{\circ} \mathrm{C}\right)$ decomposition of the 
precursor gas without using a metal catalyst ${ }^{\mathbf{1 0 , 1 1}}$ or by direct CVD growth on dielectric surfaces by simultaneously dewetting the $\mathrm{Cu}$ film. ${ }^{12}$ Even though some of these samples exhibit excellent performances, like a room-temperature Hall mobility of 2.000 $\mathrm{cm}^{2} \mathrm{~V}^{-1} \mathrm{~s}^{-1},{ }^{10}$ it is interesting to explore alternative ways of producing similar samples.

Here we report a new method to produce high quality, transparent Gr/sapphire samples in UHV. The starting point is a high-quality $\mathrm{Gr}$ layer prepared by CVD using $\mathrm{Cu}$ as a catalyst. ${ }^{13}$ The samples are then transferred to UHV, where the $\mathrm{Cu}$ film is evaporated. The samples have been characterized by helium atom scattering (HAS), Raman spectroscopy and atomic force microscopy (AFM). HAS is an established means of investigating the structure and dynamics of insulating as well as conducting surfaces in a completely nondestructive manner. ${ }^{14}$ HAS diffraction provides direct information on surface crystallinity, and HAS specular scattering is highly sensitive to surface flatness and defect density. ${ }^{15}$ Since He beams are typically $1 \mathrm{~mm}$ in size, the method provides information over a macroscopic region. Therefore, HAS is an excellent complement to Raman spectroscopy, which provides information on the quality of the Gr layer over length scales of $1 \mu \mathrm{m}$. HAS has been already used to characterize Gr grown on several metal substrates. ${ }^{\mathbf{1 6 - 1 9}}$ Our current study reports the first systematic comparison of HAS with Raman results.

Our study shows that the observation of clear He-diffraction features, which points to the existence of large Gr domains over a macroscopic region (of the order of a few microns), may be consistent with the simultaneous observation of large Raman Dpeaks arising from regions on the surface not large enough to produce diffraction peaks. Finally, our approach presents several advantages with respect to previous reported methods, like a high transparency (80\%) in the visible range, a short preparation time (30 minutes) and the use of a moderate preparation temperature $\left(\sim 900{ }^{\circ} \mathrm{C}\right)$. Finally, our method has the great advantage of being performed in UHV, leaving the $\mathrm{Gr}$ surface free of contaminants.

\section{Experimental}

\section{Sample preparation}

The starting point is a high-quality Gr layer prepared by CVD on $\mathrm{Cu}(111)$ grown on $\alpha-\mathrm{Al}_{2} \mathrm{O}_{3}(0001)$ (c-plane sapphire). The growth of $\mathrm{Cu}$ film on $c$-plane sapphire was achieved by using the electron beam evaporation method, as reported in our previous study, ${ }^{13}$ except for the total thickness $(1000 \mathrm{~nm})$ and growth rate $\left(0.3 \mathrm{~nm} \mathrm{~s}^{-1}\right)$. Different initial thicknesses of $\mathrm{Cu}(111)$ films have been used for Gr growth in order to improve the quality of the Gr layer and the elimination of $\mathrm{Cu}$ without $\mathrm{Gr}$ film deterioration. The best results have been obtained with a $1000 \mathrm{~nm}$ thick $\mathrm{Cu}$ film. For comparison, results corresponding to a sample with a lower thickness $(500 \mathrm{~nm})$ are summarized in the ESI. $\dagger$ The samples were prepared by CVD in Goettingen ${ }^{\mathbf{1 3}}$ and then transported to Madrid to complete the preparation in a UHV chamber with base pressure in the low $10^{-10}$ mbar range; typically, the samples were exposed to ambient conditions for one week before being introduced into the UHV scattering chamber for He diffraction. Once in UHV, the samples were annealed at $800 \mathrm{~K}$ with $2 \times 10^{-7}$ mbar of $\mathrm{C}_{2} \mathrm{H}_{4}$. This treatment leads to a significant improvement in surface quality, as indicated by the increase of the He-specular intensity. ${ }^{20}$ Graphene on sapphire has been obtained in situ by evaporation of the $\mathrm{Cu}$ film in UHV, heating the samples at $1150 \mathrm{~K}$ for $30 \mathrm{~min}$. The sample temperature was measured with a type-C thermocouple spot-welded to the sample holder. The sample preparation steps are schematically illustrated in Fig. 1.

\section{Results and discussion}

\section{Helium atom scattering}

The angular distributions presented in this work were measured after aligning the sample along the $\overline{\Gamma M}$ direction of the dominant Gr orientation (80\%), which corresponds to the [211] direction of the $\mathrm{Cu}(111)$ underneath. The remaining $20 \%$ of the sample is covered by domains rotated $30^{\circ}$ with respect to the $[\overline{2} 11]$ direction. $^{20}$ In the following, after discussing the shapes of specular peaks we analyze the structures corresponding to the measured diffraction peaks.

Fig. 2a shows a comparison of angular distributions of $\mathrm{He}$ atoms scattered from $\mathrm{Gr} / \mathrm{Cu} / \mathrm{Al}_{2} \mathrm{O}_{3}$ (red) and $\mathrm{Gr} / \mathrm{Al}_{2} \mathrm{O}_{3}$ (black). The high specular reflectivity and the presence of diffraction peaks demonstrate the existence of a well-ordered Gr layer, with a low density of defects, over areas of the order of the beam size (ca. $1 \mathrm{~mm}$ ). However, the width of the specular peak of $\mathrm{Gr} / \mathrm{Al}_{2} \mathrm{O}_{3}$ FWHM $=0.14^{\circ}$, which corresponds roughly to the angular resolution of the HAS machine, is lower than that of $\mathrm{Gr} / \mathrm{Cu}$ / $\mathrm{Al}_{2} \mathrm{O}_{3} \mathrm{FWHM}=0.56^{\circ}$. The width of the specular peak depends on the quality of the surface and gives information about the domain size $w$ through the relationship FWHM $1 / w^{21}$ Therefore, the above comparison can lead to the counterintuitive interpretation that the Gr layer exhibits larger domains after the copper removal. However, this is not the case as we find by comparing the surfaces of $\mathrm{Al}_{2} \mathrm{O}_{3}$ and $\mathrm{Cu} / \mathrm{Al}_{2} \mathrm{O}_{3}$. The latter has been obtained by removing the $\mathrm{Gr}$ layer in $\mathrm{Gr} / \mathrm{Cu} / \mathrm{Al}_{2} \mathrm{O}_{3}$ through $\mathrm{Ar}^{+}$sputtering, followed by annealing at $940 \mathrm{~K}$. The corresponding angular distributions are shown in the ESI (Fig. SI1 $\dagger$ ). Further heating at $1150 \mathrm{~K}$ for $30 \mathrm{~min}$ led to obtaining the clean $\mathrm{Al}_{2} \mathrm{O}_{3}$ surface. The obtained peak widths are $\mathrm{FWHM}=0.56^{\circ}$ and $0.27^{\circ}$ for $\mathrm{Cu} / \mathrm{Al}_{2} \mathrm{O}_{3}$ and $\mathrm{Al}_{2} \mathrm{O}_{3}$, respectively. This clearly shows that the FWHM of the Gr covered surface is determined mainly by the substrate underneath. The width of the specular peaks of $\mathrm{Gr} / \mathrm{Cu} / \mathrm{Al}_{2} \mathrm{O}_{3}$ and $\mathrm{Cu} / \mathrm{Al}_{2} \mathrm{O}_{3}$ are almost identical. A possible explanation is that the $\mathrm{Cu}$ film is terminated by a corrugated surface, which may induce an additional broadening of the specular peak.

On the other hand, a comparison of HAS angular distributions from $\mathrm{Al}_{2} \mathrm{O}_{3}$ and $\mathrm{Gr} / \mathrm{Al}_{2} \mathrm{O}_{3}$ (Fig. 2b) shows a decrease of the FWHM by $50 \%$ in the Gr covered surface, which points to the formation of larger domains. The inset in Fig. 2b shows the normalized specular peaks in a linear scale, where a quasitriangular background appears around the specular peaks and is especially pronounced in the $\mathrm{Al}_{2} \mathrm{O}_{3}$ spectrum. Previous HAS studies have shown that the presence of a triangular background in the vicinity of the specular peak is the signature of 

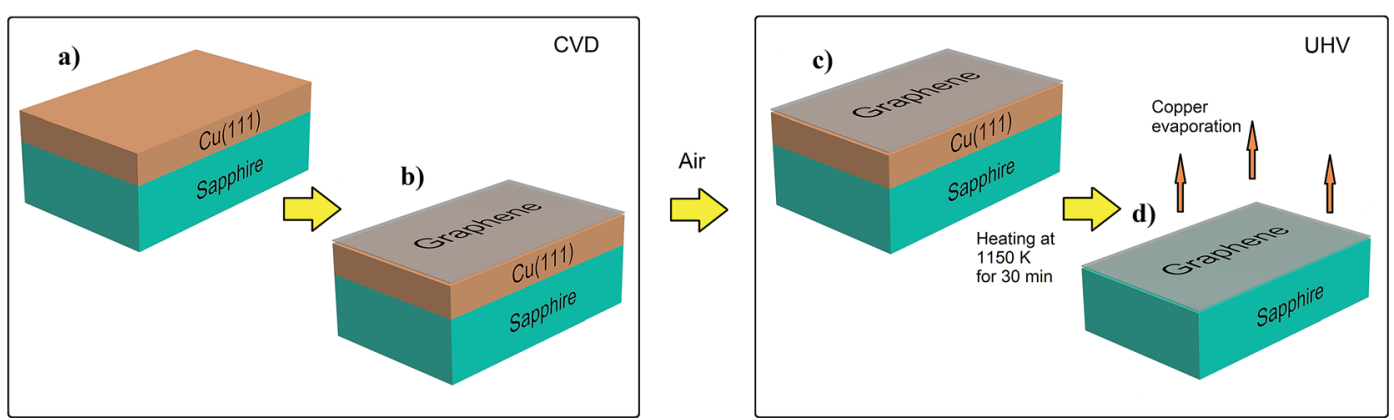

Fig. 1 Schematic procedure for the Gr growth on sapphire. (a) Copper is deposited on c-plane (0001) sapphire by electron beam evaporation. (b) Graphene growth on copper by CVD. (c) Afterexposure to ambient conditions, the sample was introduced into the He-scattering UHV chamber. (d) After heating up in UHV, Cu evaporates, leaving a Gr layer deposited on the sapphire substrate.

a random distribution of defects. ${ }^{22}$ Similar conclusions have been obtained in a more recent study reported by Manson et al. ${ }^{23}$ Therefore, we conclude that scattering from point defects dominates in both surfaces, although to a lesser degree in the $\mathrm{Gr}$ covered surface. These observations suggest that the Gr layer covers like a carpet the rough and flat sapphire substrate, hiding a portion of the single defects and softening the roughness of the step edges. Even though the Gr layer certainly contains many defects, our data suggest that the $\mathrm{Gr} / \mathrm{Al}_{2} \mathrm{O}_{3}$ layer exhibits a reduced number of defects, leading to an average domain size larger than that of clean $\mathrm{Al}_{2} \mathrm{O}_{3}$. Since the FWHM observed for $\mathrm{Gr} / \mathrm{Al}_{2} \mathrm{O}_{3}$ is limited by the resolution of our system, we can conclude that the average domain size is at least one order of magnitude larger than the transfer width of our system $(200 \mathrm{~nm})$. The coherence length for the $\mathrm{Al}_{2} \mathrm{O}_{3}$ surface, which is of the same order of the size ${ }^{24}$ of the terraces is $\sim 40 \mathrm{~nm}$. The measured absolute reflectivity of $\mathrm{He}$ atoms from $\mathrm{Gr} / \mathrm{Al}_{2} \mathrm{O}_{3}$ is $5 \%$ at the incident conditions used in Fig. 2b. This high reflectivity combined with the narrow specular peak observed suggests that these samples are excellent candidates to be used as a focussing mirror in scanning He atom microscopy. ${ }^{25-27}$

The different diffraction peaks in the angular distributions of Fig. 2 correspond to the periodicities of the Gr, sapphire and multiple moire superstructures resulting from the mismatch of the two surfaces. The lattice constant of $\mathrm{Gr}$ on sapphire has been determined from the positions of its first order diffraction peaks indicated as $G(0,1)$ and $G(\overline{1}, 0)$ in Fig. $2 a$ and $b$, using the in-plane Bragg diffraction condition for a hexagonal twodimensional structure. The derived value $a=(2.44 \pm 0.02) \AA$ is identical to the one reported for $\mathrm{Gr} / \mathrm{Cu}(111),{ }^{20}$ and agrees well with $2.4612 \AA$, the periodicity of a single carbon layer in graphite. ${ }^{28}$ The low intensity of the first order diffraction peaks points to a low corrugation of the $\mathrm{Gr} / \mathrm{Al}_{2} \mathrm{O}_{3}$ surface. Wellresolved and intense first order diffraction peaks are present in the blue spectrum in Fig. $2 \mathrm{~b}$ of the $\mathrm{Al}_{2} \mathrm{O}_{3}$ substrate, denoted by $\mathrm{Al}_{2} \mathrm{O}_{3}(10)$ and $\mathrm{Al}_{2} \mathrm{O}_{3}(\overline{1} 0)$, appear at the positions expected for the shortest hexagonal lattice length of sapphire (Al-Al distance), $a=2.74 \AA$ ․ As far as we know, this is the first Hediffraction spectrum reported from a clean sapphire surface.

The He-diffraction spectrum measured from the $\mathrm{Gr} / \mathrm{Al}_{2} \mathrm{O}_{3}$ surface exhibits a more complex structure, due to the moiré reconstruction. The (10) diffraction peak of the atomic lattice of $\mathrm{Gr}$ (denoted by $\operatorname{Gr}(1,0)$ ) is observed at $23^{\circ}$ away from the specular peak $(0,0)$. However, the existence of other diffraction peaks, due to the moiré superstructure, is clearly detected along the whole diffraction pattern. The angular positions of the diffraction peaks from the Gr moiré lattice are in good agreement with expected values for a hexagonal structure with a periodicity of $30 \AA$. In particular, the third, the sixth and the eleventh order diffraction of the moire pattern (labelled $\mathrm{m}(3,0)$, $\mathrm{m}(6,0)$ and $\mathrm{m}(11,0))$ are clearly observed in the black spectrum in Fig. 2b. A comparison of the angular positions of diffraction peaks of $\mathrm{Gr} / \mathrm{Al}_{2} \mathrm{O}_{3}$ and clean $\mathrm{Al}_{2} \mathrm{O}_{3}$ surfaces shows that the highintensity sharp peak $\mathrm{m}(11,0)$ matches exactly the position of the peak $(1,0)$ of sapphire. Thus, we can conclude that the moiré pattern corresponds to a $(11 \times 11)$ periodicity aligned with the $(1 \times 1)$ sapphire unit cell. This is schematically shown in Fig. $3 \mathrm{~b}$. The moiré structure has been investigated by varying the azimuthal orientation of the sample. Fig. 3a shows several He-diffraction angular distributions. Each spectrum has been recorded after a small azimuthal rotation of the sample with respect to an axis lying in the incident plane. The black curve is the same as that shown in Fig. 2 and is denoted as $0^{\circ}$. Rotating the sample we can see how different peaks from the moiré pattern are detected. In the red curve, rotated by $0.5^{\circ}$, the peak $\mathrm{m}(11,0)$ disappears, whereas the $\mathrm{m}(9,0)$ and the $\mathrm{m}(10,0)$ diffraction peaks are clearly resolved. With a further rotation of $0.5^{\circ}$ (denoted as $1^{\circ}$, blue curve), the measured spectrum exhibits weaker but still clear peaks for different order of diffraction $(\mathrm{m}(8,0), \mathrm{m}(4,0)$ and $\mathrm{m}(2,0))$. For a rotation of $1.5^{\circ}$ (magenta curve) the peaks $\mathrm{m}(4,0)$ and $\mathrm{m}(2,0)$ are still observed, but the $\mathrm{m}(8,0)$ peak is not present. The green curve has been recorded for an azimuthal rotation of $\sim 30^{\circ}$. The position of the detected diffraction peaks fits the diffraction pattern produced by a $\mathrm{Gr}$ layer rotated by $30^{\circ}$ with respect to the $(1 \times 1)$ sapphire unit cell, which corresponds to a $(\sqrt{3} \times \sqrt{3}) \mathrm{R} 30^{\circ}$ structure. The presence of shadows of the $\mathrm{m}(1,0)$ and $\mathrm{m}(2,0)$ peaks in this spectrum is due to small misalignment of the tilt angle which appears for different azimuthal orientations.

The diffraction patterns from the moire superstructure indicate that the $\mathrm{Gr} / \mathrm{sapphire}$ system keeps the same domain ratio $\left(80 \%\right.$ along the main direction, and $20 \%$ rotated $30^{\circ}$ with 

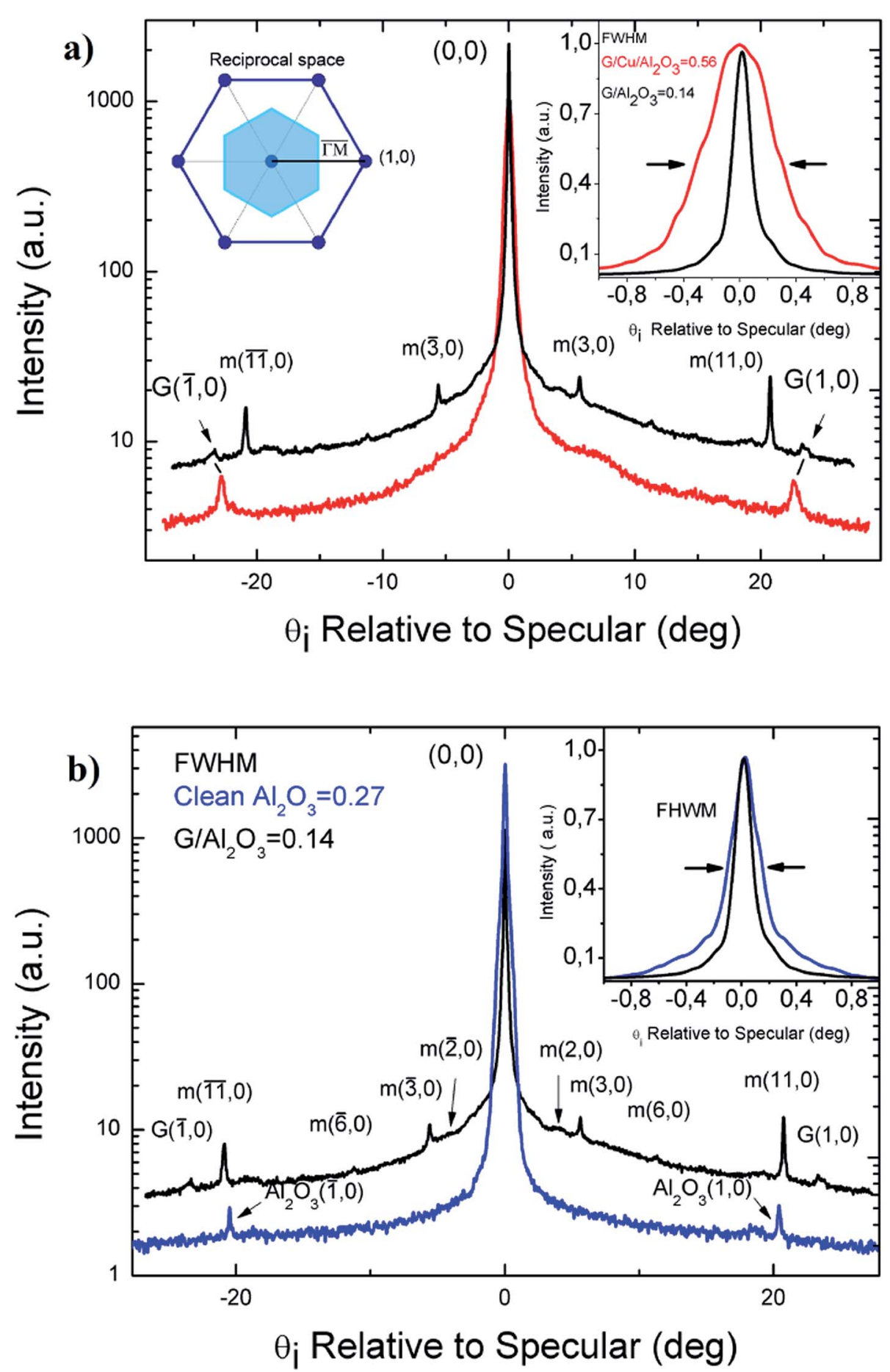

Fig. 2 (a) Angular distributions of $\mathrm{He}$ atoms scattered from $\mathrm{Gr} / \mathrm{Cu} / \mathrm{Al}_{2} \mathrm{O}_{3}$ (red spectrum) and $\mathrm{Gr} / \mathrm{Al}_{2} \mathrm{O}_{3}$ (black spectrum) along $\overline{\Gamma M}$. (b) Comparison of $\mathrm{He}$ diffraction spectra from $\mathrm{Gr} / \mathrm{Al}_{2} \mathrm{O}_{3}$ (black) and clean $\mathrm{Al}_{2} \mathrm{O}_{3}$ (blue). The sample temperature is $90 \mathrm{~K}$, and the He-incident energy is $E_{\mathrm{i}}=19.8$ $\mathrm{meV}$ (the small shift in the position of first order peaks is a result of quite small differences in the He beam energy). The inset on the right side shows a detailed view of the profile of the specular peaks. The diffraction peaks of the moire superstructure are labelled as " $m(n, 0)$ ".

respect to this direction) present in the starting system, $\mathrm{Gr} / \mathrm{Cu} /$ $\mathrm{Al}_{2} \mathrm{O}_{3}$. We have also recorded He-diffraction spectra for intermediate azimuthal rotations, and no evidence of additional periodicities has been detected. It is interesting to compare our data with the results reported by Hwang et al. ${ }^{10}$ These authors have grown Gr on $c$-plane (0001) sapphire directly without metal catalysis and concluded, based on detailed spatial electron diffraction measurements and DFT calculations, that Gr on sapphire grows forming a single dominant crystal orientation for around $80 \%$ of the material. In their work, however, the orientation is determined at an early stage of the nucleation during the growth process, and therefore multiple rotational 

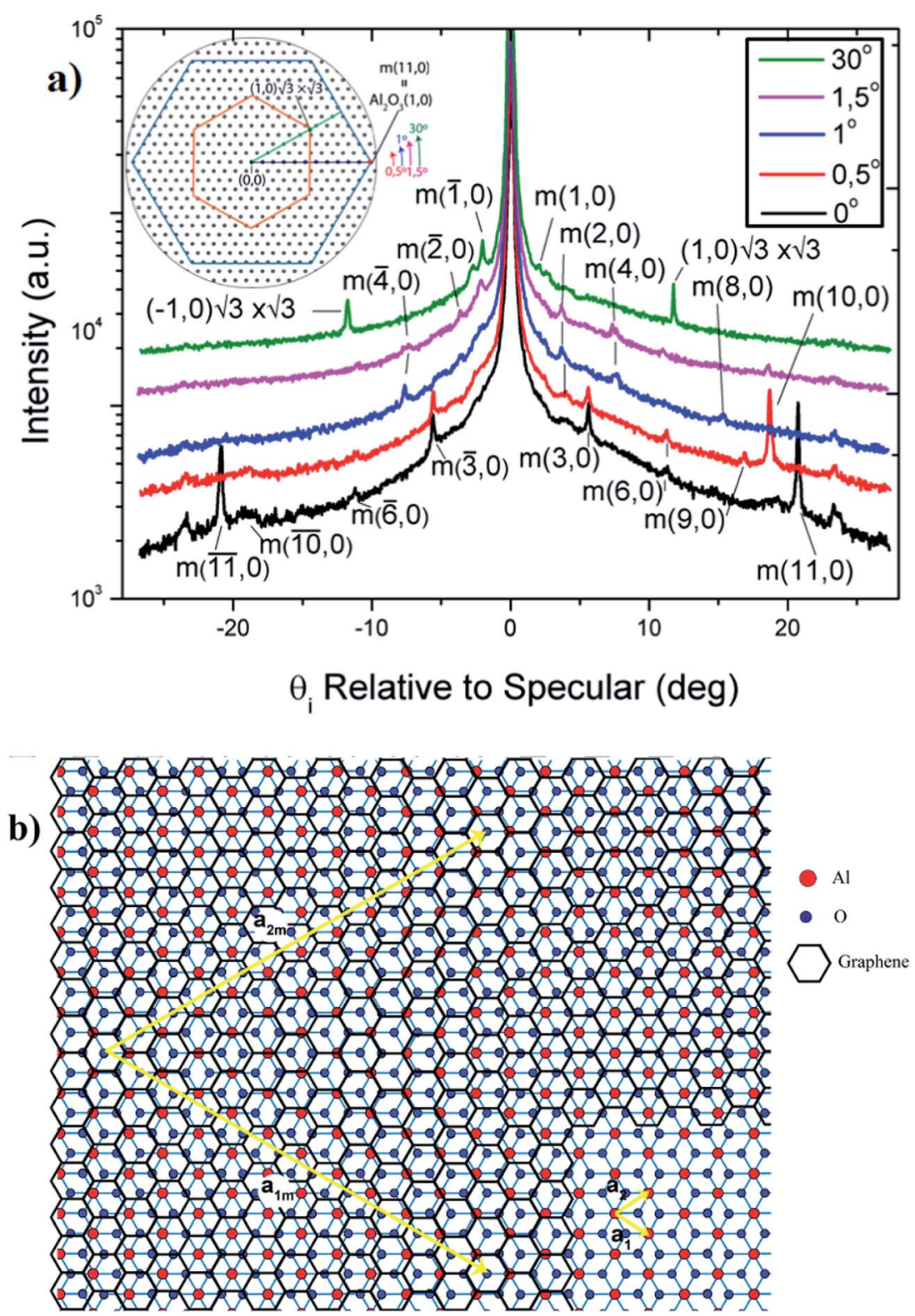

Fig. 3 (a) Angular distribution of He scattering from $\mathrm{Gr} / \mathrm{Al}_{2} \mathrm{O}_{3}$ as a function of azimuthal rotation. The inset shows the directions on the moiré reciprocal lattice and the first order diffraction of the sapphire (blue hexagon) and $(\sqrt{3} \times \sqrt{3}) R 30^{\circ}$ (red hexagon) structures. (b) Proposed model for the moiré $(11 \times 11)$ structure, which is obtained by superimposing $(12 \times 12)$ Gr honeycombs on top of $(11 \times 11)$ sapphire unit cells. The primitive vectors of the moiré $\left(a_{1 m}\right.$ and $\left.a_{2 m}\right)$ and sapphire unit cells $\left(a_{1}\right.$ and $\left.a_{2}\right)$ are also shown.

domains with respect to the substrate are obtained. The preparation method described in our current work, on the other hand, could in principle lead to the formation of only one rotational domain if the starting point, i.e. $\mathrm{Gr} / \mathrm{Cu} / \mathrm{Al}_{2} \mathrm{O}_{3}$, was formed by only one domain.

Hwang et al. also performed DFT calculations (including the van der Waals interaction) for commensurate cells with orientations of $0^{\circ}, 11^{\circ}$ and $22^{\circ}$ with respect to the sapphire. ${ }^{10}$ From the similarity of the binding energy obtained for all three commensurate cells it has been concluded that the dispersion interaction depends weakly on the orientation of the Gr layer on the sapphire surface. The unit cells rotated by $11^{\circ}$ and $22^{\circ}$ with respect to the sapphire correspond actually to moiré patterns with a periodicity smaller than $(11 \times 11)$, and are therefore not consistent with our HAS data. However, our data provide evidence for the presence of the cell with orientation of $0^{\circ}$, which corresponds to the above mentioned $(\sqrt{3} \times \sqrt{3}) \mathrm{R} 30^{\circ}$ structure.

\section{Phonon dispersion}

Several series of time-of-flight (TOF) spectra have been taken under different incident conditions, in order to get the phonon dispersion curves. A representative series of TOF data is shown in Fig. SI2. $\dagger$ Fig. 4 shows the phonon dispersion curves for Gr on sapphire measured along the $\overline{T M}$ direction. Phonon dispersion 


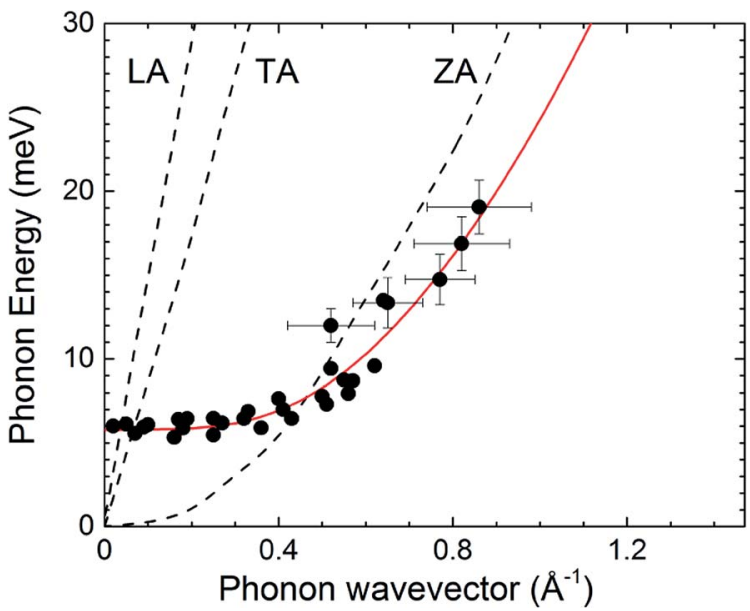

Fig. 4 Experimentally derived surface phonons for $\mathrm{Gr}$ on sapphire measured along the $\overline{T M}$ direction shown with DFT calculations for free standing $\mathrm{Gr}^{29}$ (dashed curves). Error bars are shown for some data points. Red curve is a fit to the data - see text.

curves for free standing $\mathrm{Gr}$ calculated from first principles ${ }^{29}$ are also shown as dashed lines. No data points were detected for the in-plane longitudinal acoustic (LA) and transverse acoustic (TA) modes. The TA mode is usually forbidden in HAS measurements for planar scattering in the $\overline{\Gamma M}$ direction due to symmetry as has been shown for $\mathrm{Gr} / \mathrm{Cu}(111),{ }^{20}$ but this selection rule is not valid in the presence of defects that break the translational symmetry ${ }^{30}$ However, the small energy difference between the LA and TA mode makes it very hard to resolve them in the TOF measurements. We therefore could not obtain any information on these two modes here. The out-of-plane acoustic (ZA) mode, on the other hand, is clearly resolved and as expected from the broken symmetry introduced by the sapphire substrate, does not follow a pure acoustic dispersion but has a frequency $\omega_{0}=5.8 \mathrm{meV}$ at the $\Gamma$ point. The ZA mode exhibits a parabolic dispersion, softened with respect to the one expected for free standing Gr. This softening is related to the presence of point defects in $\mathrm{Gr}$, as shown below.

The ZA dispersion curve can be used to derive more fundamental quantities like the Gr-sapphire coupling strength, $g$, and the free standing bending rigidity $\kappa .{ }^{31}$ As mentioned above, coupling Gr to a substrate will introduce a gap at a frequency $\omega_{0}$ at the $\bar{T}$ point, and the dispersion relation of the flexural mode $\mathrm{ZA}$ is given by: ${ }^{32,33}$

$$
\omega_{\mathrm{ZA}}^{\text {coupled }}(\Delta K)=\sqrt{\frac{\kappa}{\rho_{2 \mathrm{D}}} \Delta K^{4}+\omega_{0}^{2}},
$$

where $\rho=7.6 \times 10^{-8} \mathrm{~g} \mathrm{~cm}^{-2}$ is the two-dimensional mass density of $\mathrm{Gr}, \omega_{0}=\sqrt{g / \rho_{2 \mathrm{D}}}$ and $g$ is the coupling strength between $\mathrm{Gr}$ and substrate. ${ }^{32}$ Therefore, from a fit to the experimental data using eqn (1) (red curve in Fig. 4) it is possible to determine both $g$ and $\kappa$. We obtain $g=(5.8 \pm 0.4) \times 10^{19} \mathrm{~N} \mathrm{~m}^{-3}$ for the $\mathrm{Gr}$-sapphire interaction. This value is almost identical to the one reported for $\mathrm{Gr}-\mathrm{Cu}\left(g=(5.7 \pm 0.4) \times 10^{19} \mathrm{~N} \mathrm{~m}^{-3}\right)$ applying the same procedure. ${ }^{20}$ However, we get $\kappa=0.61 \pm 0.15$ $\mathrm{eV}$ for $\mathrm{Gr}-$ sapphire, quite below the value reported for $\mathrm{Gr}-\mathrm{Cu}$ $(\kappa=1.30 \pm 0.15 \mathrm{eV})$ and for free standing $\mathrm{Gr}(\kappa=1.20-1.61 \mathrm{eV}$ (ref. 31)). This low value of $\kappa$ reflects the softening of the ZA mode with respect to the one of free standing $\mathrm{Gr}$, and points to a lower Young's modulus of $\mathrm{Gr}$ on sapphire. A recent study has shown that the elastic modulus of Gr decreases with a higher density of vacancies in the Gr lattice.$^{34}$ Extrapolation of the data presented in ref. 34 shows that a reduction of the Young's modulus by a factor of 2 can be induced by $2 \%$ vacancies. Thus, our results can be interpreted as due to the presence of additional defects in $\mathrm{Gr} / \mathrm{sapphire}$ (as compared to $\mathrm{Gr} / \mathrm{Cu}$ ), which might arise during the sample preparation through $\mathrm{Cu}$ evaporation in vacuum.

\section{AFM results}

Atomic force microscopy confirms the presence of Gr domains over lateral scales of microns in the metal-free regions between $\mathrm{Cu}$ microislands $(0.5-1 \mu \mathrm{m}$ height, $0.5-5 \mu \mathrm{m}$ lateral diameter, based on optical and atomic force microscopy scrutiny) formed after $\mathrm{Cu}$ evaporation. AFM characterization is focused on the metal-free areas, as shown in Fig. 5. AFM topographic images show the presence of large Gr-covered areas (a substrate coverage ranging $55-80 \%$ was estimated from the analysis of several regions of the sample, as illustrated in ESI, Fig. SI $3 \dagger$ ). Graphene flakes reach 1-3 $\mu \mathrm{m}$ size; actually, it seems that some Gr flakes of approximately $3 \mu \mathrm{m}$ size suspended on the sapphire substrate are apparently torn into smaller flakes. By comparison of this sample with the one with initial thickness of $500 \mathrm{~nm} \mathrm{Cu}$ (see Fig. SI 4 ), we show that the increasing of the thickness leads to a wider Gr coverage. A profile analysis revealed that the Gr layers on the sapphire substrate have a height of 6-8 $\AA$, in agreement with the thickness of monolayer $\mathrm{Gr}^{12}$ showing randomly oriented wrinkles of 1-4 $\mathrm{nm}$ size (see height profiles in Fig. 5a and b). Actually, root mean-square (rms) roughness of the Gr layers averaged from randomly selected flakes is $5.3 \AA$, visibly rougher than the smooth single crystal sapphire substrate, which exhibits an rms roughness of $2.2 \AA$ An illustration of the rms roughness study is depicted in the ESI file as Fig. SI5. $\dagger$ Moreover, residual $\mathrm{Cu}$ nanometric grains are observed at the boundaries of the Gr domains, presenting an average size of $2-4 \mathrm{~nm}$, as illustrated in the height profiles (Fig. 5a).

The substrate reveals a wavy morphology, attributed to the presence of terraces (see Fig. 5b) typical of (0001) single crystal sapphire. ${ }^{10}$ The height of the steps ranges between 4 and $5 \AA$ (i.e. around $c / 3$, where $c=12.99 \AA$ is the lattice constant of $c$-plane sapphire), and its periodicity ranges between 100-150 nm. However, an estimation of the coherence length obtained from the width of the specular HAS peak, that gives an average terrace $\operatorname{size}^{24}$ of $40 \mathrm{~nm}$, is smaller than the terrace periodicity measured with AFM. This is consistent with the high sensitivity of HAS to low densities of defects, which leads in average to the detection of smaller terraces. Moreover, the sample exhibits the peculiar morphology of the substrate. From the AFM images in the dynamic mode, the presence of nanometric pits decorating the sapphire substrate is corroborated, predominantly located at the Gr flake boundaries, not present in the bare substrate areas (see Fig. SI6†). 

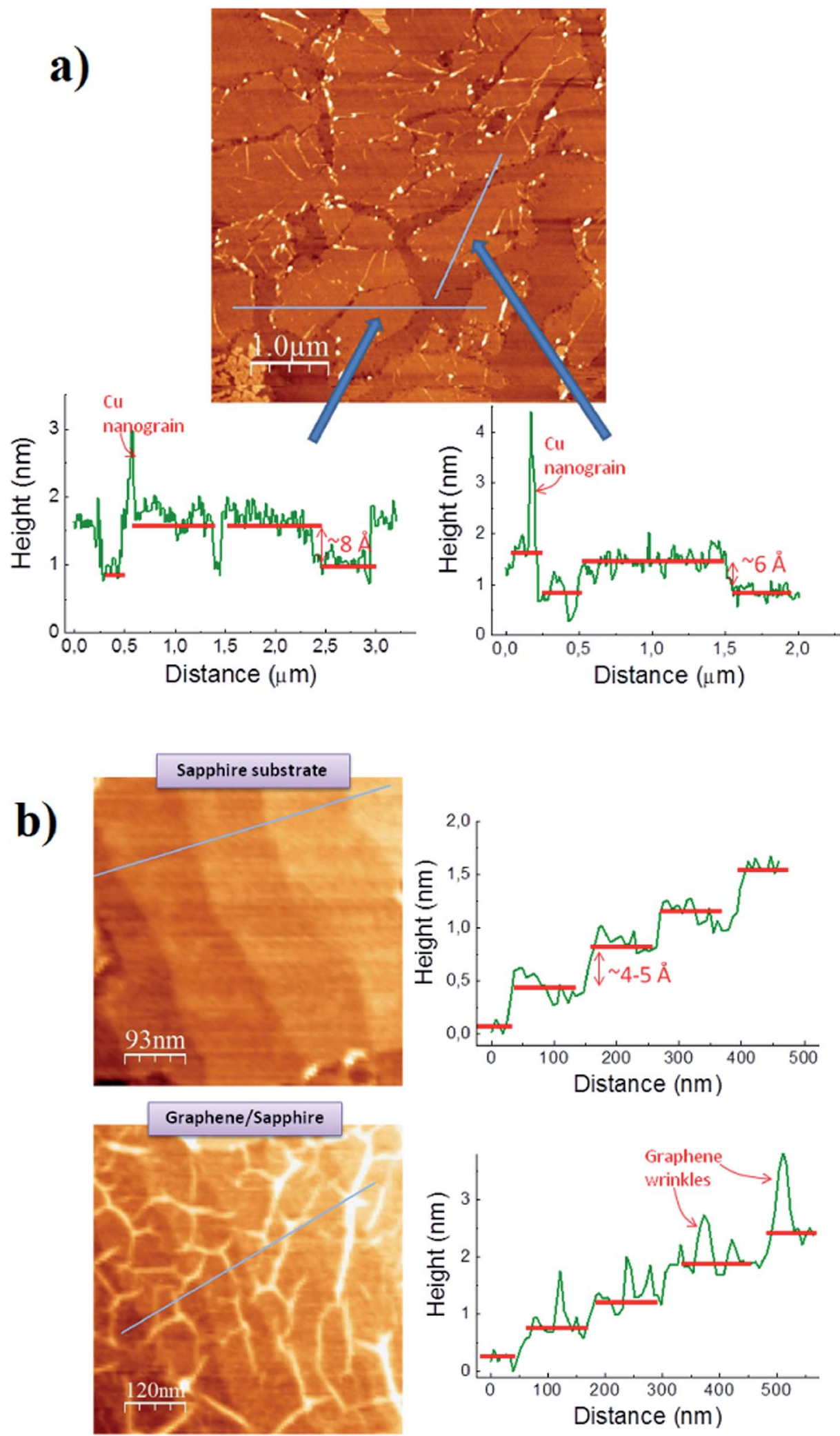

Fig. 5 (a) AFM topographic image of the Gr/sapphire sample (image: $5 \mu \mathrm{m} \times 5 \mu \mathrm{m}$, trying to get an appropriate vision of the Gr flake dimensions). AFM image and height profile analysis reveal discontinuous Gr layers of size up to $3 \mu \mathrm{m}$, with $\sim 6-8 \AA$ height. (b) AFM images of another region showing zoomed areas representative of the sapphire substrate and $\mathrm{Gr} /$ sapphire, with respective height profiles, illustrating the presence of steps corresponding to the substrate and how Gr accommodates to the substrate surface. 
To gain insight into the possible presence of nanometric pits, we have carried out an additional AFM experiment in the contact mode, which in principle would require a lower spring constant (range $0.1-1 \mathrm{~N} \mathrm{~m}^{-1}$ ), ${ }^{35,36}$ using a tip adequate for the dynamic mode with a stronger spring constant (10-100 $\mathrm{N} \mathrm{m}^{-1}$ ). Such a strong spring constant of the tip eventually removed the Gr layers, allowing extra topological information and revealing differences between the bare substrate and the substrate underneath the Gr flakes. Contact mode images (Fig. SI6†) confirm the presence of a pit array originally located at the $\mathrm{Gr}$ boundary, likely determining the Gr dimensions (depth 1-2 nm; width variable from 20 to $100 \mathrm{~nm}$ ). Also, some nanometric pits were observed, to a lesser extent, in the regions where originally Gr settled. The origin of these nanometric pits in the sapphire substrate is likely due to $\mathrm{Al}_{2} \mathrm{O}_{3}$ carbothermal reduction in vacuum. ${ }^{10,37}$ It is suggested that the tearing process of the $\mathrm{Gr}$ layer can be attributed to an effect of such substrate carbothermal reduction combined with the breakthrough of the $\mathrm{Gr}$ layer by the evaporated copper.

\section{Raman spectroscopy}

Optical transmission and Raman micro-spectroscopy were also used to characterize the Gr before and after the elimination of the copper film for a film thickness of $1000 \mathrm{~nm} \mathrm{Cu}$. The Raman spectra indicate that single layer graphene is found all over the sample in all cases (Fig. SI7 of the ESI $\dagger$ ). AFM measurements indicate that the height of the Gr flakes with respect to the substrate is in the range 6-8 $\AA$, which is consistent with single layer graphene. The optical image in Fig. 6a shows the remaining sparse $\mathrm{Cu}$ microislands after $\mathrm{Cu}$ evaporation. The optical transmission for a $4 \mathrm{~mm}$ diameter area after annealing at $1150 \mathrm{~K}$ is around $80 \%$ and the characteristic transition at 267 $\mathrm{nm}$ reveals the presence of Gr (Fig. 6b). We ascribe the weak feature around $580 \mathrm{~nm}$ to the plasmon of remaining $\mathrm{Cu}$ microstructures, which can also be seen in the optical (Fig. 6a) and AFM images (Fig. 5a). The quotient of the intensities of the characteristic Gr G and 2D Raman peaks, $I_{2 \mathrm{D}} / I_{\mathrm{G}}$, is related to the quality of the Gr domains, that is, the regularity of the carbon network. For values close to that of pristine Gr the ratio decrease is related to the presence of strain fields and doping. ${ }^{38,39}$ At a high concentration of defects which involves breaking a large fraction of the $\mathrm{sp}^{2} \mathrm{C}=\mathrm{C}$ bonds by the formation of vacancies, irregular rings such as Stone Wales defects or $\mathrm{O}, \mathrm{OH}-$ or other functional groups, the $I_{2 \mathrm{D}} / I_{\mathrm{G}}$ ratio decreases very significantly and the width of all Raman peaks increases. ${ }^{40}$ The so-called defects peak D (TO mode close to the $\bar{K}$ point of the Brillouin zone) is activated by the presence of defects that supply the required momentum for the Raman process. Therefore, both $I_{2 \mathrm{D}} / I_{\mathrm{G}}$ and $I_{\mathrm{D}} / I_{\mathrm{G}}$ ratios are used to evaluate the Gr quality. Graphene in the as deposited samples is of high quality, with a typical $I_{2 \mathrm{D}} / I_{\mathrm{G}}$ ratio for $\mathrm{Gr}$ on copper and no defects peak $\mathrm{D}$, as shown in Fig. SI7. $\dagger$ After annealing Gr to $1150 \mathrm{~K}$, the $I_{2 \mathrm{D}} / I_{\mathrm{G}}$ ratio is very similar to the as deposited sample but the defect peak clearly increases (Fig. SI7 from ESI† and Fig. $6 \mathrm{~g}$ and $\mathrm{h}$ ). This has been observed for $\mathrm{Gr}$ on remaining $\mathrm{Cu}$ regions and in areas where $\mathrm{Cu}$ has been completely eliminated.
The situation is analogous for Gr deposited on 500 and 1000 $\mathrm{nm} \mathrm{Cu}$ films. Raman images of $\mathrm{Gr} /$ sapphire have been measured to obtain a statistical analysis of the relevant parameters. The optical image of a $10 \times 10 \mu \mathrm{m}^{2}$ area of the sample is replicated by the Raman image of the background due to $\mathrm{Cu}$ (2000-2500 $\mathrm{cm}^{-1}$ region) (Fig. 6c and d) allowing us to distinguish $\mathrm{Gr} / \mathrm{Cu}$ and $\mathrm{Gr} /$ sapphire regions (Fig. $6 \mathrm{~g}$ and $\mathrm{h}$ ). This area is representative of the measured Raman images. The image obtained from the $I_{2 \mathrm{D}} / I_{\mathrm{G}}$ intensity ratio (Fig. 6f) evidences the uniformity of Gr over the sample and its quality in terms of the regularity of the carbon network, which is similar to the asdeposited Gr. On the contrary, the $I_{\mathrm{D}} / I_{\mathrm{G}}$ image indicates that the density of defects on sapphire is higher that on the remaining $\mathrm{Cu}$ regions (Fig. 6e). The intensity of the $\mathrm{D}$ peak is a compromise between an enhancing factor, the formation of defects that allows the Raman process, and a depleting factor which is the reduction of the density of carbon aromatic rings. The $\mathrm{D}$ mode corresponds to a vibration of the aromatic rings while $\mathrm{G}$ vibration only requires $\mathrm{sp}^{2}$ hybridization of carbon, therefore $\mathrm{G}$ peak intensity is much less sensitive to the presence of defects. The $I_{\mathrm{D}} / I_{\mathrm{G}}$ ratio is used to estimate the density of point defects $(0 \mathrm{D}$ defects) or the size of Gr grains (1D defects) by using different formulas. Both situations present similar behavior upon increasing the defect density or reducing the Gr size: the $I_{\mathrm{D}} / I_{\mathrm{G}}$ ratio first increases up to a maximum value that depends on the excitation wavelength (stage I) and then decreases (stage II). Deciding which kind of defect (0D or 1D) is predominant is not straightforward in most cases. The pioneering work of Tuinstra and Koening (TK) ${ }^{\mathbf{4 1}}$ on nanocrystalline graphite established that $I_{\mathrm{D}} / I_{\mathrm{G}}=C / L_{\mathrm{a}}$, where $L_{\mathrm{a}}$ is the graphite crystallite size and $C$ depends on the laser excitation wavelength. This dependence was established to be $C(\lambda)(\mathrm{nm})=2.4 \times 10^{-10} \lambda^{4}(\mathrm{~nm})^{42}$ (stage I) but below around $2-3 \mathrm{~nm}$ there is a second regime (stage II) where the ratio decreases as the crystallite size is reduced $I_{\mathrm{D}} /$ $I_{\mathrm{G}}=C^{\prime} L_{\mathrm{a}}{ }^{2} \cdot{ }^{43}$ The constant $C^{\prime}$ is obtained by imposing continuity between both stages resulting in our case $C^{\prime} \sim 0.438$ for $\lambda_{\text {laser }}=$ $488 \mathrm{~nm}$. On the other hand, the controlled ion bombardment of $\mathrm{Gr}$ with different doses to produce carbon vacancies has led to the formulation of a phenomenological model that correlates $I_{\mathrm{D}} / I_{\mathrm{G}}$ and the distance between the defects, $L_{\mathrm{D}} \cdot{ }^{43}$ The functional dependences of $L_{\mathrm{a}}$ (black lines) and $L_{\mathrm{D}}$ (red circles) versus $I_{\mathrm{D}} / I_{\mathrm{G}}$ are plotted in Fig. 7a for $\lambda_{\text {laser }}=488 \mathrm{~nm}$.

Fig. 7b shows the number of events of the $I_{\mathrm{D}} / I_{\mathrm{G}}$ values obtained in the Raman image of Fig. 6e. The distribution is wide and reaches $I_{\mathrm{D}} / I_{\mathrm{G}}$ ratio up to 4 . Since both $L_{\mathrm{a}}$ and $L_{\mathrm{D}}$ are bivaluated, to know whether Gr is in stage I or II we have to look at the widths of the peaks and at the $I_{2 \mathrm{D}} / I_{\mathrm{G}}$ values. The narrowness of the peaks and the high $I_{2 \mathrm{D}} / I_{\mathrm{G}}$ ratio ensure that Gr is in stage I in spite of the very high $I_{\mathrm{D}} / I_{\mathrm{G}}$ values. The wide distribution of Fig. 7a can be explained to be due to Gr domains with size $L_{\mathrm{D}}$ from 14 to $3 \mathrm{~nm}$ (following the black curve in stage I) or to a combination of large regions with point defects with distances $L_{\mathrm{D}}=3$ to $9 \mathrm{~nm}$ (from the curve with red circles) for the lower $I_{\mathrm{D}} /$ $I_{\mathrm{G}}$ values up to around 2.9 and small Gr domains in the range from 3 to $5 \mathrm{~nm}$ for $2.9<I_{\mathrm{D}} / I_{\mathrm{G}}<4$. These two scenarios cannot be differentiated by Raman spectroscopy alone; however, the information provided by $\mathrm{He}$ diffraction indicates that large 
a)

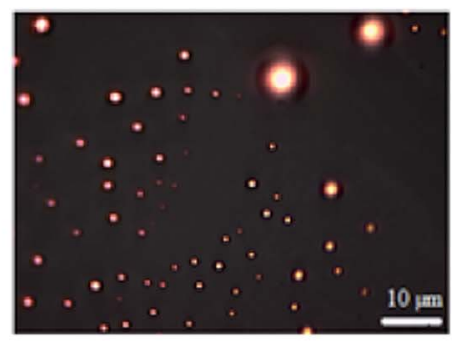

c)

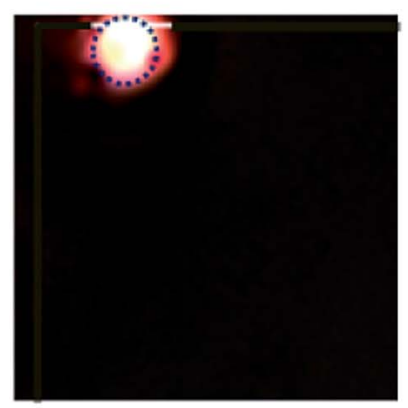

d)
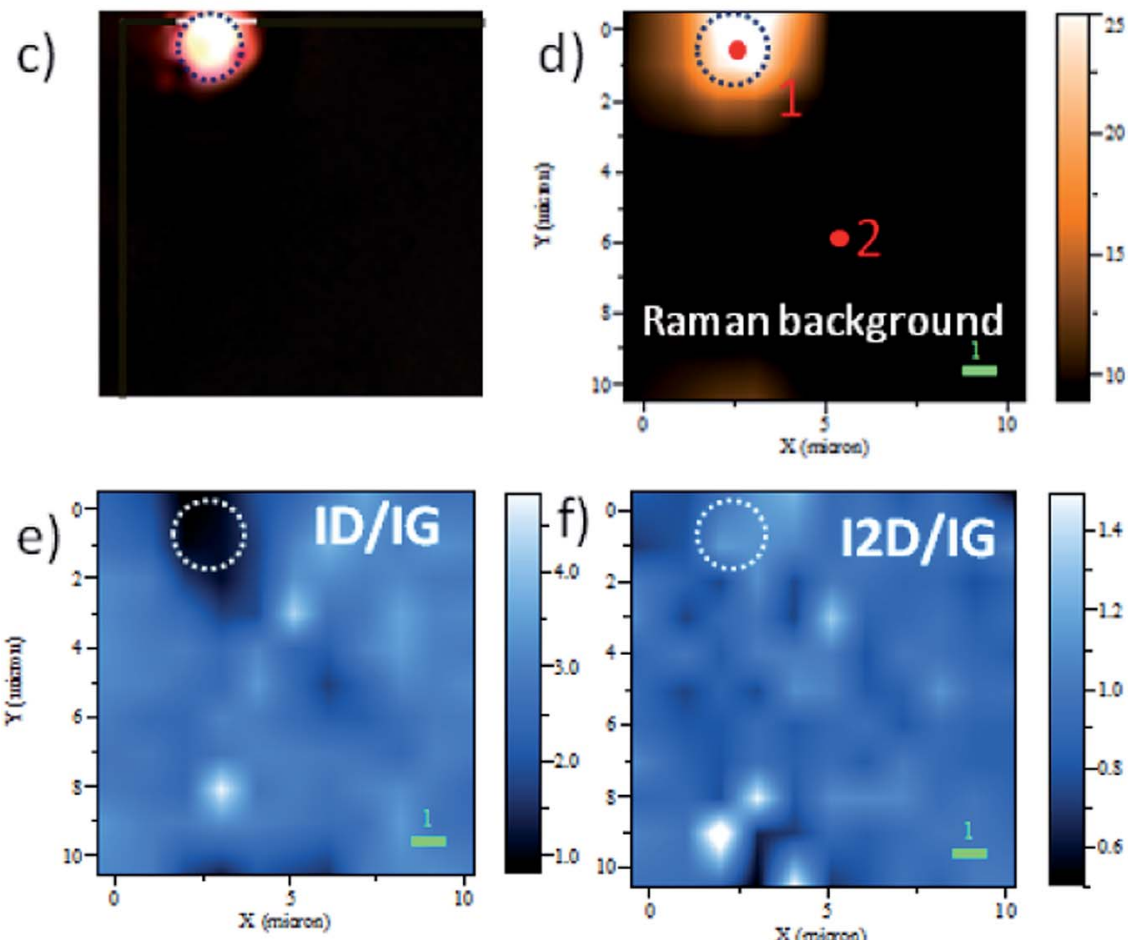

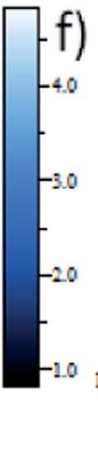

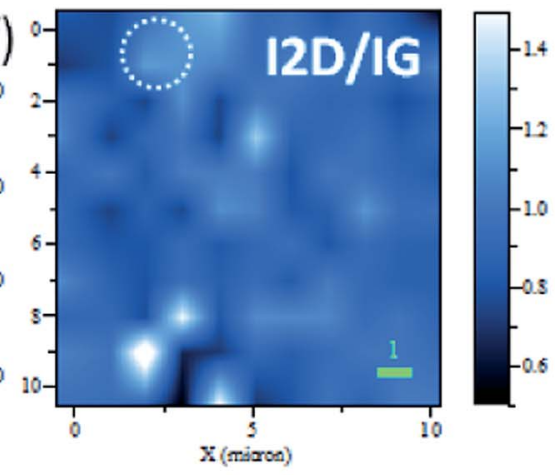
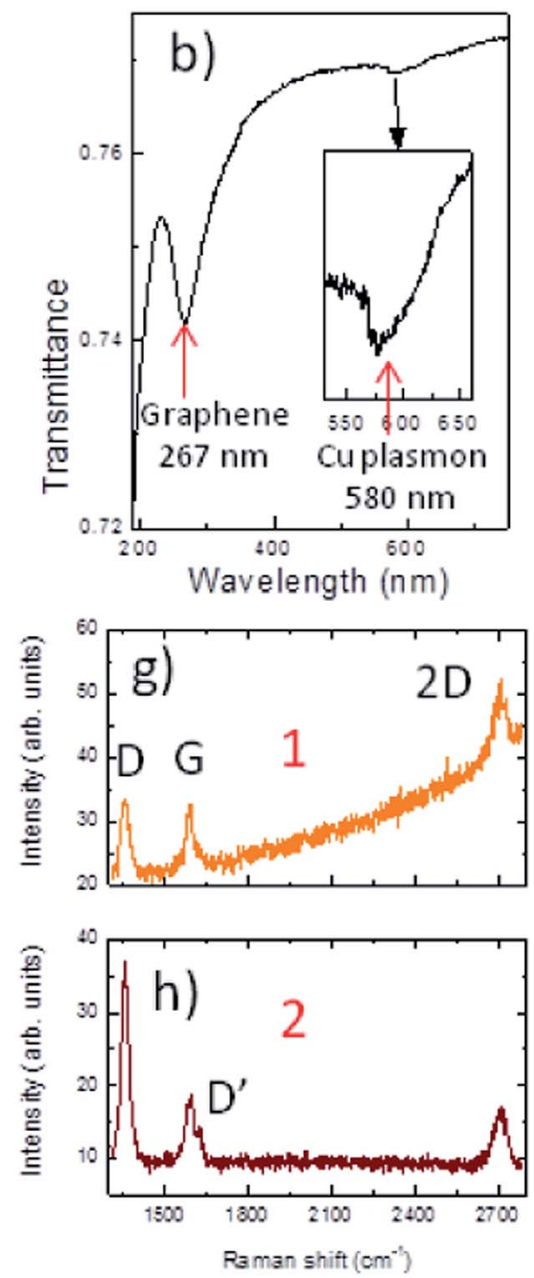

Fig. 6 (a) Optical images with Cu residues (scale bar $10 \mu \mathrm{m}$ ). (b) Optical transmittance in a 4 mm diameter area. The interband Gr transition and Cu plasmon (a zoom is shown in the inset) are indicated. (c) Optical image, (d) Raman image $\left(10 \times 10 \mu \mathrm{m}^{2}\right)$ of the background revealing the Cu residues. (e) $I_{D} / I_{G}$ and (f) $I_{2 D} / I_{G}$ Raman images. Raman spectra of Gr on sapphire sample (g) and on Cu (or remaining Cu microstructures) (h). D, G and 2D characteristic peaks of $\mathrm{Gr}$ are indicated.

coherent domains are present therefore discarding the first case. The $0 \mathrm{D}$ defects density can be then calculated for the $I_{\mathrm{D}} / I_{\mathrm{G}}$ $<2.9$ using $n_{\mathrm{D}}\left(\mathrm{cm}^{-2}\right)=10^{14} / \pi L_{\mathrm{D}}{ }^{2}$ (ref. 43 and 44) obtaining $n_{\mathrm{D}}=3 \times 10^{13}$ to $3 \times 10^{12} \mathrm{~cm}^{-2}$. Since the density of carbon atoms in $\mathrm{Gr}$ is $3.9 \times 10^{15} \mathrm{~cm}^{-2}$, the estimated defect fractions, from $0.7 \%$ to $7 \%$, are below the threshold for the reduction of He diffraction intensity.

\section{Discussion}

An important message from our study is that the wrong conclusion might be drawn if both HAS and Raman results were not available. The observation of clear diffraction peaks, including a moiré pattern, suggests the existence of a wellordered Gr overlayer, with little presence of defects, whereas the observation of such a pronounced D peak in the Raman spectra points to a Gr layer broken into nanometric patches or grains. It should be kept in mind that the two techniques probe different length-scales and are sensitive to different aspects of Gr. While HAS probes macroscopic regions (mm), Raman microscopy averages information from areas $\sim 0.7 \mu \mathrm{m}$ in diameter. Most importantly, the He-diffraction signal originates from the less defective regions while the Raman D peak originates exclusively from the defects of Gr (red areas in Fig. 8). Therefore, these techniques reveal quite different aspects of the same samples. Nevertheless, the high intensity of the HAS diffraction peak indicates its origin from a large fraction of the sample and the hundreds of Raman spectra collected across several $10 \mu \mathrm{m} \times 10 \mu \mathrm{m}$ areas present small variations. Therefore, the combination of both sets of data proves the existence of large domains of $\mathrm{Gr}$ with localized defects as carbon vacancies or arrays of vacancies probably originating during the $\mathrm{Cu}$ evaporation at the wrinkles or defects present in the initial $\mathrm{Gr}$ layer grown on the $\mathrm{Cu}$ film.

The average domain size of $\mathrm{Gr} / \mathrm{sapphire}$ derived from HAS is $2000 \mathrm{~nm}$, in good agreement with the Gr size obtained from 


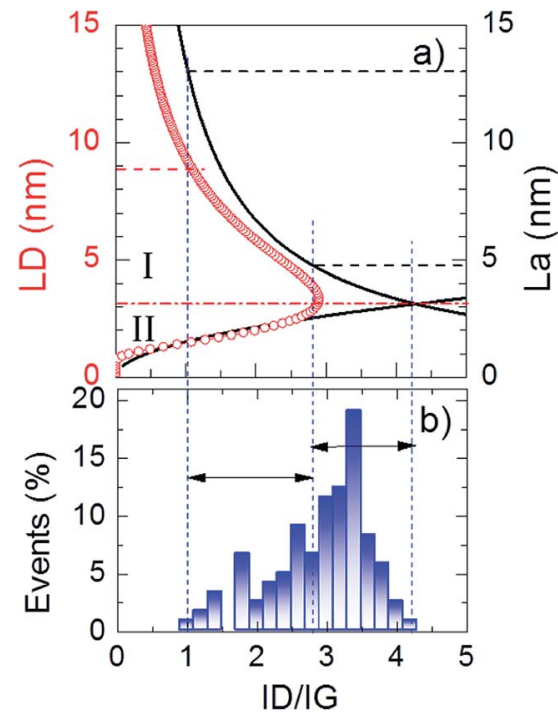

Fig. 7 (a) $L_{a}$ (black lines) and $L_{D}$ (red circles) versus $I_{D} / I_{G}$ for $\lambda_{\text {laser }}=488$ $\mathrm{nm}$; (b) statistical distribution of $I_{D} / I_{G}$ values in the Raman image of Fig. 6e.

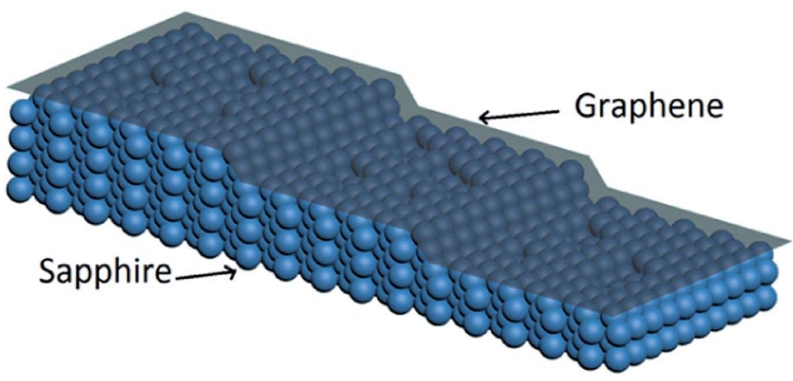

Top view

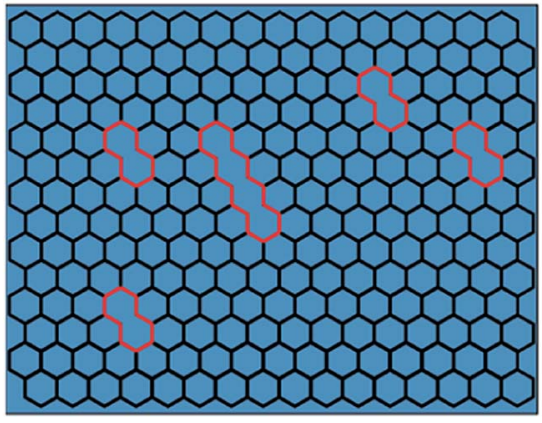

Fig. 8 Simplified view of the model proposed to explain both HAS and Raman results of $\mathrm{Gr}$ on sapphire. The top view shows a surface area leading to He-diffraction. The red areas represent regions leading to the appearance of Raman D signal (Gr defects). See text for details.

AFM data. For the underlying sapphire substrate, AFM images show terraces (4-5 A height) with an average domain size of 120 $\mathrm{nm}$, much larger than the $40 \mathrm{~nm}$ obtained for the sapphire substrate from the specular width in HAS (which sets a lower limit to the domain size). The different values obtained with AFM and HAS for the average domain size of sapphire are a consequence of the different interaction mechanisms involved in both techniques. Thermal energy He atoms interact with the surface at a very low electron density ( $c a .10^{-4}$ a.u.), 2-3 $\AA$ above the surface atoms. This interaction is characterized by a large cross section to single defects. Thus, defects on sapphire reduce the domain size as seen by HAS, leading to the detection of smaller terraces on average as compared to AFM. The larger domain size observed for $\mathrm{Gr} /$ sapphire is a consequence of a similar mechanism. The Gr layer covers the sapphire substrate like a carpet (see Fig. 8), hiding defects and steps, leading to the appearance of larger coherently diffracting domains. This mechanism can be deduced from the strong reduction of the specular FWHM for Gr/sapphire as compared to the sapphire substrate.

The high sensitivity of HAS to point defects, combined with the Debye-Waller attenuation of diffraction intensities, makes it impossible to detect diffraction from surfaces as soon as the density of defects is larger than $15-20 \%$. This explains for instance the absence of diffraction features from sapphire substrates prepared from scratch, in situ, in our laboratory. Thus, from the observation of high specular reflectivity and diffraction peaks from $\mathrm{Gr} / \mathrm{sapphire,} \mathrm{we} \mathrm{can} \mathrm{conclude} \mathrm{that} \mathrm{the}$ density of point defects must be near $5 \%$. This is consistent with the estimation made from the Raman data ( 0.7 to $7 \%$ ) and the $2 \%$ derived from the determination of the bending rigidity by phonon measurements. The almost complete elimination of $\mathrm{Cu}$ is attained without any significant deterioration of the Gr film. This is possible due to the strong planar $\mathrm{C}-\mathrm{C}$ bond in comparison with the weak $\mathrm{Gr}-\mathrm{Cu}$ interaction. Presumably, the copper film leaves the substrate through defects and borders of the $\mathrm{Gr}$ patches as indicated by the detection of ultra-small $\mathrm{Cu}$ particles (around 2-3 nm) at these borders (Fig. 5a).

\section{Conclusions}

We have shown that a high-quality Gr layer on sapphire can be grown by heating in UHV a Gr layer prepared by CVD on $\mathrm{Cu}(111) / \mathrm{Al}_{2} \mathrm{O}_{3}$. The heating process leads to evaporation of the Cu film, leaving a Gr layer deposited on the sapphire substrate. These samples require a short preparation time (30 minutes) and moderate heating temperatures $\left(\sim 900{ }^{\circ} \mathrm{C}\right)$. Since the method is performed in UHV, it keeps the Gr surface free of contaminants. These samples present a high transparency $(80 \%)$ in the visible range, making them suitable for many applications. Raman mapping shows that the $\mathrm{G}$ to $2 \mathrm{D}$ peak ratio is comparable for both $\mathrm{Gr} / \mathrm{Cu} / \mathrm{Al}_{2} \mathrm{O}_{3}$ and $\mathrm{Gr} / \mathrm{Al}_{2} \mathrm{O}_{3}$ samples, suggesting that the final quality is mainly limited by the quality of the starting Gr layer. HAS and AFM data prove the presence of flat and large Gr domains over lateral scales of microns. Hediffraction data reveal that $\mathrm{Gr}$ forms an $(11 \times 11)$ moiré pattern, aligned with the $(1 \times 1)$ sapphire unit cell. The bending rigidity $k=0.61 \pm 0.15 \mathrm{eV}$ and the $\mathrm{Gr}$-sapphire coupling strength $g=(5.8 \pm 0.4) \times 10^{19} \mathrm{~N} \mathrm{~m}^{-3}$ have been determined from HAS data of the phonon dispersion of the $\mathrm{Gr}$ flexural mode. Our study shows that HAS and Raman provide complementary information on the sample's quality, and that the wrong conclusion might be drawn if only one of these techniques is applied. 


\section{Acknowledgements}

This project was sponsored by the European Union, Seventh Framework Programme: Theme NMP.2012.1.4-3 Grant no. 309672. AMW and HKY acknowledge support from the Alexander von Humboldt Foundation, the Max Planck EPFL Center for Molecular Nanoscience and Technology as well as the Deutsche Forschungsgemeinschaft under CRC 1073. Additionally, a part of the research has been supported by the Spanish MINECO, under project MAT2012-37276-C03-01, and by Comunidad de Madrid, under project S2013/MIT-2740 (PHAMA_2.0-CM).

\section{References}

1 K. Ellmer, Nat. Photonics, 2012, 6, 809.

2 D. Wei and J. Kivioja, Nanoscale, 2013, 5, 10108.

3 F. Bonaccorso, L. Colombo, G. Yu, M. Stoller, V. Tozzini, A. C. Ferrari, R. S. Ruoff and V. Pellegrini, Science, 2015, 347, 1246501.

4 D. Lee, H. Lee, Y. Ahn, Y. Jeong, D.-Y. Leeb and Y. Lee, Nanoscale, 2013, 5, 7750.

5 X. Li, W. Cai, J. An, S. Kim, J. Nah, D. Yang, R. Piner, A. Velamakanni, I. Jung, E. Tutuc, S. K. Banerjee, L. Colombo and R. S. Ruoff, Science, 2009, 324(5932), 1312-1314.

6 Y. Lee, S. Bae, H. Jang, S. Jang, S. E. Zhu, S. H. Sim, Y. I. Song, B. H. Hong and J. H. Ahn, Nano Lett., 2010, 10(2), 490-493.

7 S. Bhaviripudi, X. Jia, M. S. Dresselhaus and J. Kong, Nano Lett., 2010, 10(10), 4128-4133.

8 S. Bae, et al., Nat. Nanotechnol., 2010, 5, 574-578.

9 H. Ago, Y. Ogawa, M. Tsuji, S. Mizuno and H. Hibino, J. Phys. Chem. Lett., 2012, 3(16), 2228-2236.

10 J. Hwang, M. Kim, D. Campbell, H. a. Alsalman, J. Y. Kwak, S. Shivaraman, A. R. Woll, A. K. Singh, R. G. Hennig, S. Gorantla, M. H. Rümmeli and M. G. Spencer, ACS Nano, 2013, 7, 385-395.

11 M. A. Fanton, J. A. Robinson, C. Puls, Y. Liu, M. J. Hollander, B. E. Weiland, M. LaBella, K. Trumbull, R. Kasarda, C. Howsare, et al., ACS Nano, 2011, 5, 8062-8069.

12 A. Ismach, C. Druzgalski, S. Penwell, A. Schwartzberg, M. Zheng, A. Javey, J. Bokor and Y. Zhang, Nano Lett., 2010, 10, 1542-1548.

13 H. K. Yu, K. Balasubramanian, K. Kim, J. L. Lee, M. Maiti, C. Ropers, J. Krieg, K. Kern and A. M. Wodtke, ACS Nano, 2014, 8, 8636-8643.

14 D. Farías and K. H. Rieder, Rep. Prog. Phys., 1998, 61, 15751664.

15 B. Poelsema and G. Comsa, Scattering of Thermal Energy Atoms from Disordered Surfaces, Springer, Berlin, 1989.

16 B. Borca, S. Barja, M. Garnica, M. Minniti, A. Politano, J. M. Rodriguez-García, J. J. Hinarejos, D. Farías, A. L. Vázquez de Parga and R. Miranda, New J. Phys., 2010, 12, 093018.

17 A. Politano, B. Borca, M. Minniti, J. J. Hinarejos, A. L. Vázquez de Parga, D. Farías and R. Miranda, Phys. Rev. B: Condens. Matter Mater. Phys., 2011, 84, 23.

18 H. Shichibe, Y. Satake, K. Watanabe, A. Kinjyo, A. Kunihara, Y. Yamada, M. Sasaki, W. W. Hayes and J. R. Manson, Phys. Rev. B: Condens. Matter Mater. Phys., 2015, 91, 155403.
19 A. Tamtögl, E. Bahn, J. Zhu, P. Fouquet, J. Ellis and W. Allison, J. Phys. Chem. C, 2015, 119, 25983.

20 A. Al Taleb, H. K. Yu, G. Anemone, D. Farías and A. M. Wodtke, Carbon, 2015, 95, 731-737.

21 G. Comsa, Surf. Sci., 1979, 81(1), 56-68.

22 W. A. Schlup and K. H. Rieder, Phys. Rev. Lett., 1986, 56, 73.

23 F. Hofmann, J. P. Toennies and J. R. Manson, J. Chem. Phys., 1994, 101, 10155.

24 J. Lapujoulade, Y. Lejay and G. Armand, Surf. Sci., 1980, 95, 107-130.

25 B. Holst and W. Allison, Nature, 1997, 390, 244.

26 K. Fladischer, H. Reingruber, T. Reisinger, V. Mayrhofer, W. E. Ernst, A. E. Ross, D. A. MacLaren, W. Allison, D. Litwin and J. Galas, New J. Phys., 2010, 12, 033018.

27 D. Barredo, F. Calleja, P. Nieto, J. J. Hinarejos, G. Laurent, A. L. Vázquez de Parga, D. Farías and R. Miranda, Adv. Mater., 2008, 20, 3492-3497.

28 J. B. Nelson and D. P. Riley, Proc. Phys. Soc., 1945, 57, 477.

29 J. A. Yan, W. Y. Ruan and M. Y. Chou, Phys. Rev. B: Condens. Matter Mater. Phys., 2008, 77, 125401.

30 F. de Juan, A. Politano, G. Chiarello and H. A. Fertig, Carbon, 2015, 85, 225-232.

31 Y. Wei, B. Wang, J. Wu, R. Yang and M. L. Dunn, Nano Lett., 2013, 13, 26-30.

32 B. Amorin and F. Guinea, Phys. Rev. B: Condens. Matter Mater. Phys., 2013, 88, 115418.

33 A. Al Taleb and D. Farías, J. Phys.: Condens. Matter, 2016, 28, 103005.

34 G. Lopez-Polin, C. Gómez-Navarro, V. Parente, F. Guinea, M.-I. Katsnelson, F. Pérez-Murano and J. Gómez-Herrero, Nat. Phys., 2014, 11, 26-31.

35 A. J. Marsden, M. Phillips and N. R. Wilson, Nanotechnology, 2013, 24, 255704.

36 M. Halmann, A. Steinfeld, M. Epstein and I. Vishnevetsky, Miner. Process. Extr. Metall. Rev., 2014, 35, 126-135.

37 F. Jiménez-Villacorta, E. Climent-Pascual, R. RamírezJiménez, J. Sánchez-Marcos, C. Prieto and A. de Andrés, Carbon, 2016, 101, 305.

38 M. Kalbac, A. Reina-Cecco, H. Farhat, J. Kong, L. Kavan and M. S. Dresselhaus, ACS Nano, 2010, 4, 6055-6063.

39 X. Díez-Betriu, S. Álvarez-García, C. Botas, P. Álvarez, J. Sánchez-Marcos, C. Prieto, R. Menéndez and A. de Andrés, J. Mater. Chem. C, 2013, 1, 6905.

40 F. Tuinstra and J. L. Koenig, J. Chem. Phys., 1970, 53, 11261130.

41 L. G. Cançado, K. Takai, T. Enoki, M. Endo, Y. a. Kim, H. Mizusaki, A. Jorio, L. N. Coelho, R. Magalhães-Paniago and M. A. Pimenta, Appl. Phys. Lett., 2006, 88, 1-4.

42 A. Ferrari and J. Robertson, Phys. Rev. B: Condens. Matter Mater. Phys., 2000, 61, 14095.

43 M. M. Lucchese, F. Stavale, E. H. M. Ferreira, C. Vilani, M. V. O. Moutinho, R. B. Capaz, C. A. Achete and A. Jorio, Carbon, 2010, 48, 1592-1597.

44 A. C. Ferrari and D. M. Basko, Nat. Nanotechnol., 2013, 8, 235-246. 